\title{
Upsurge of genital Chlamydia trachomatis infection
}

Background and epidemiology: Since 1997 the rate of genital Chlamydia trachomatis infection in Canada has increased by more than $60 \%$, up from 114 per 100000 population in 1997 to 188 per 100000 in 2003 (www.hc-sc.gc.ca/pphbdgspsp/std-mts/stddata_pre06_04 /tab1-1_e.html). This discouraging trend may be due in part to improved detection methods, such as the introduction of noninvasive urine testing and nucleic acid amplification techniques. The upsurge is consistent with national trends observed for other bacterial STDs such as gonorrhea and syphilis. National rates of gonorrhea increased by more than 50\% between 1997 and 2002, and those of infectious syphilis were almost 3 times higher in 2002 than in $1997 .{ }^{1}$ The trend renders Health Canada's national goal of 50 cases of genital chlamydial infection per 100000 by 2010 a diminishing hope.

Age and sex are the greatest risk factors for genital chlamydial infection; rates are highest among females aged 15-24 years $(1380$ per 100000$)$ and males aged 20-24 years (608 per 100 000). Genital C. trachomatis is transmitted through direct genital-genital or genital-anal contact. Transmission through direct orogenital contact is possible, although rare. Pharyngeal infection is thought to be mild and transient. Although symptoms of genital C. trachomatis infection usually begin 1 to 3 weeks after infection, up to $50 \%$ of infected men and $70 \%$ of infected women have no symptoms. Infection can persist for years undetected and untreated. Asymptomatic carriers act as the के main reservoir for spread.

Symptomatic infection in women is characterized by pelvic pain, burning on urination and abnormal vaginal bleeding. Examination reveals a mucopurulent endocervix that may appear edematous, erythematous and friable owing to inflammation of the endocervical columnar epithelium. Less frequent manifestations include bartholinitis, urethral syndrome with dysuria and pyuria, perihepatitis (FitzHugh-Curtis syndrome) and proctitis. Infection during pregnancy may result in premature rupture of membranes and preterm delivery, and conjunctival and pneumonic infection of the newborn. ${ }^{2}$ If left untreated, C. trachomatis infection in women can progress to pelvic inflammatory disease, chronic pelvic pain and ectopic pregnancy. Symptoms in men can include a faint, watery discharge from the penis, urethral itch and burning on urination. Complications include epididymitis (and possible infertility) and Reiter's syndrome. Homosexual receptive intercourse may result in proctitis.

Clinical management: In general, annual screening of sexually active women aged 25 and younger is recommended. ${ }^{3}$ Screening should also be offered routinely at STD clinics and to sexually active patients with risk factors (see list at right). It may also be prudent to screen older women at less frequent intervals (e.g., at the time of their regular Pap test).

A complete clinical evaluation involves a genital and pelvic examination, including a swab of secretions from the urethra (male) or cervix (female). Testing urine is a good option for people for whom a genital examination is impractical or unacceptable. Optimal urine samples are those from patients who have not urinated for 2 hours and include the first $10 \mathrm{~mL}$ collected. Most Canadian laboratories offer nucleic acid amplification testing using polymerase chain reaction, which has better sensitivity and specificity than the traditional enzyme immunoassay $(94 \%-$ $99 \%$ and $98 \%-100 \%$ v. $60 \%-$ $65 \%$ and $75 \%-95 \%$ respectively). People who engage in oral or anal sex may benefit by having these sites swabbed and the specimens sent for culture.

The treatment of choice for uncomplicated $C$. trachomatis infection of the cervix, anus or urethra is $1 \mathrm{~g}$ of azithromycin orally in 1 dose. Alternative regimens include $100 \mathrm{mg}$ of doxycycline orally twice daily for 7 days or $300 \mathrm{mg}$ of ofloxacin orally twice daily for 7 days. Infected people should abstain from sexual intercourse for 7 days after starting treatment. In general, retesting for the possibility of therapeutic failure is not recommended unless symptoms persist, the woman is pregnant or there is good reason to believe therapy has failed. Retesting should not be conducted before 3 weeks after initiating treatment because of the risk of a false-positive result. Everyone who has received treatment for C. trachomatis infection should be screened again within the year after treatment because of the risk of reinfection.

Cases of $C$. trachomatis infection should be reported to the local public health unit. The most recent sexual contact and all sexual partners within 60 days of the date of diagnosis of the index case should be notified and tested. Health unit staff can assist with this.

Prevention: The use of condoms provides the best protection.

\section{Erica Weir}

Associate Medical Officer of Health

Kingston, Frontenac and Lennox

\& Addington Health Unit

Kingston, Ont.

\section{References}

1. 2002 Canadian sexually transmitted infections (STI) surveillance report: pre-release. Ottawa: Health Canada; 2002. Available: www.hc-sc.gc.ca/pphbdgspsp/std-mts/stddata_pre06_04/index.html (accessed 2004 Sept 13).

2. Steben M. The resurgence of Cblamydia trachomatis. 7 Obstet Gynaecol Can 2004;26(6):552-9.

3. US Centers for Disease Control and Prevention. Sexually transmitted diseases treatment guidelines 2002. MMWR Recomm Rep 2002;51(RR-6):1-78.
Sexually active people who should be offered screening for genital Chlamydia trachomatis infection

- Women $\leq 25$ years old

- Men who have sex with men

- Anyone with a new sexual partner in the past 2 months

- Anyone with more than 2 sexual partners in the past year

- Street youths

- Anyone with a history of an STD in the past year

-Women requesting an abortion

- Sex trade workers

- Anyone who has recently travelled to a country where STDs and HIV infection are highly endemic or who has a sexual partner who has done so

- Anyone who requests an STD screen 